WA MX:

\title{
THE FEBRUARY 5, 1965 SOLAR PROTON EVENT: 2. LOW ENERGY PROTON OBSERVATIONS AND THEIR RELATION TO THE MAGNETOSPHERE
}

D. J. WILLIAMS

C. O. BOSTROM

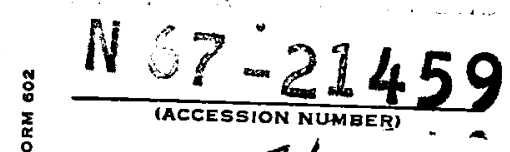

$\frac{\text { (ACCESSION NUMEEB) }}{\text { NASA TAGES }}$

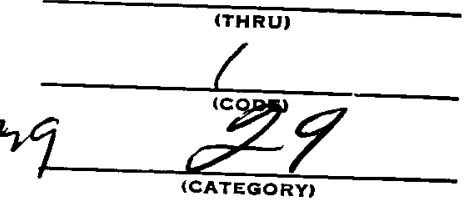

JANUARY 1967 
The February 5, 1965 Solar Proton Event:

2. Low Energy Proton Observations and Their Relation to the Magnetosphere

\author{
by \\ D. J. Williams \\ Goddard Space Flight Center
}

and

C. O. Bostrom

The Johns Hopkins University

Applied Physics Laboratory

January 1967

NASA/Goddard Space Flight Center, Greenbelt, Maryland 


\begin{abstract}
Observations of the temporal and spatial behavior of low energy ( $\sim 1 \mathrm{Mev})$ solar protons are presented for the solar proton event of February 5, 1965, utilizing data from the near earth satellites 1963 38C and INJUN 4 and spaceprobe Mariner 4. An enhancement of low energy proton intensities is observed at invariant latitudes $\Lambda \gtrsim 80^{\circ}$ and may be a low altitude effect of the high latitude topology of the magnetopause. Comparison of the polar cap and Mariner 4 observations indicate that the magnetospheric configuration varies as the boundary conditions imposed by the interplanetary medium vary, thereby causing marked structure in the polar cap proton intensities. Further comparisons of the polar cap and Mariner 4 observations are presented and discussed in terms of (1) a filamentary interplanetary field structure and (2) an extended geomagnetic tail. Depending on the assumptions either approach is consistent with the data. It is also indicated that particle motion across interplanetary field lines near the solar surface or in the region of the shock front is required to explain the low energy proton arrival times in the vicinity of the earth.
\end{abstract}




\section{INTRODUCTION}

The preceeding paper (Bostrom et al, 1967) has discussed observations of polar cap protons obtained from satellite 1963 38C during the Feruary 5, 1965 solar proton event. It was noted there that the low energy ( $\sim 1 \mathrm{Mev}$ ) portion of the proton spectrum displayed a temporal history more complex than that of the high energy $(\gtrsim 10 \mathrm{Mev})$ portion of the spectrum and that the behavior of the low energy protons did not appear consistent with particle diffusion.

In this note we shall consider in more detail the behavior of the low energy ( 1 Mev) protons observed during the February 5, 1965 event. Additional low energy proton data from satellite INJUN 4 and spaceprobe Mariner 4 have been very generously made available to us by Drs. S. M. Krimigis and J. A. Van Allen of the University of Iowa and are further discussed in a companion paper (Krimigis and Van Allen, 1967)

The main points to be emphasized here are:

1. The observation of an enhancement of low energy polar cap protons at latitudes (a) which may be connected to a high latitude neutral line on the magnetopause or (b) which may represent the first geomagnetic interconnected field line;

2. The observation that rapid or direct access of low energy protons to polar cap regions appears to be alternately allowed and denied, implying a magnetospheric configuration which varies in concert with the changing boundary conditions imposed by the interplanetary medium; and

3. The comparison of the low energy proton time history as observed over the polar cap and as observed at the position of Mariner 4, some $3700 \mathrm{R}_{\mathrm{E}}$ away from the earth in nearly the anti-solar direction. 
DATA AND RESULTS

Polar Cap Data: Details of the detectors, satellites and orbits have been presented elsewhere (Van Allen, 1965; Van Allen and Krimigis, 1965; Krimigis and Van A1len, 1965, 1967; Whelpley, 1965; Williams and Smith, 1965; Bostrom et a1, 1967). Therefore these items shall only be briefly summarized in the following paragraphs.

The magnetically oriented satellite 1963 38C was launched on September 28, 1963 into a nearly circular polar orbit having a $1147 \mathrm{~km}$ apogee, a $1067 \mathrm{~km}$ perigee, an $89.9^{\circ}$ inclination and a 107.5 minute period. At the time of the February 5, 1965 solar proton event, the orbital plane of 1963 38C made an angle of $53^{\circ}$ with the earth sun 1 ine. Data during the event were obtained from the dayside hemisphere only (local time of $\sim 1530$ hours).

The two proton spectrometers aboard 1963 38C were oriented to look out normal to and along the alignment axis. This yielded, after alignment, look directions oriented normal $\left(90^{\circ}\right)$ to the local line of force and, in the northern hemisphere, up $\left(180^{\circ}\right)$ the line of force (away from the earth). Each of the proton spectrometers consists of two 500 micron $(\mu)$ thick surface barrier solid state detectors arranged in a telescope mount. The proton differential energy windows sampled are $1.2-2.2 \mathrm{Mev}$, $2.2-8.2 \mathrm{Mev}, 8.2-25 \mathrm{Mev}$, and 25 - $100 \mathrm{Mev}$. The 1963 38C data from the February 5, 1965 event have been corrected for a cosmic ray background and a high energy omnidirectional proton background (Bostrom et a1, 1967) 
Satellite INJUN 4, also magnetically oriented, was launched on November 21, 1964 into an orbit having a $2502 \mathrm{~km}$ apogee, a $527 \mathrm{~km}$ perigee and an $81^{\circ}$ inclination (Whelpley, 1965). A thin (25 $\mu$ ) surface barrier solid state detector with two discriminator levels was used to obtain proton energy intervals of $0.52-4.2 \mathrm{Mev}$ and $0.90-2.1 \mathrm{Mev}$ (Krimigis and Van Allen, 1967). During the February 5, 1965 event, INJUN 4 data were obtained from local times of 0500 - 0915 hours.

Figure 1 presents the time history of the low energy polar cap protons during the period February 5-8, 1965 as obtained from the $1.2-2.2 \mathrm{Mev}$ channel of $196338 \mathrm{C}$ and the $0.9-2.1 \mathrm{Mev}$ channel of INJUN 4. The data points are polar cap averages for $L \geq 10 \mathrm{R}_{\mathrm{E}}$ in the case of $196338 \mathrm{C}$ and for $\mathrm{L} \geq 8 \mathrm{R}_{\mathrm{E}}$ in the case of INJUN 4 and have been normalized using the 1963 38C pass of February 6, 1647 UT and the INJUN 4 pass of February 6, 1637 UT. Absolute flux comparisons and geometric factor uncertainties are discussed in Bostrom et al (1967). From their Table 2, the above two proton channels agree to better than a factor of 1.7 in absolute flux. The February 5, 1965 flare is also shown in Figure 1. The flare occurred at a solar disc heliocentric position of $25^{\circ} \mathrm{W}, 8^{\cup} \mathrm{N}$, began at $1750 \mathrm{UT}$, reached maximum phase at 1810 UT and ended at 2010 UT (Solar-Geophysical Data, 1965). The sudden commencement shown occurred at $\sim 1414$ UT February 6, 1965(Lincoln, 1965).

It is seen that the $196338 \mathrm{C}$ and INJUN 4 data seem to be in good agreement except for the rise to the initial peak in intensity ( 0600 UT, February 6) of these low energy protons. This rise is characterized 
by a rather marked temporal structure. Subsequent comparisons with Mariner 4 show that such structure is absent at the position of Mariner 4. This has led us to inspect the polar cap data for possible discrepancies with the result that none have been found and that the observed structure appears to be real. Note that the structure occurring during this early phase of the event is observable within the INJUN 4 data itself.

Figure 2 presents the normalized time history of low energy polar cap protons during the February 5 event using the $0.52-4.2 \mathrm{Mev}$ channe 1 of INJUN 4 and $1.2-2.2$ Mev channe1 of 1963 38C. These data have been normalized using the 1963 38C pass of February 6, 2208 UT and the INJUN 4 passes of February 6, 2123 UT and 2228 UT. Figure 2 shows that a similar, complex time structure is also seen when the 1963 $38 \mathrm{C}$ data is normalized to this lower energy channel. Figures 1 and 2 also show an apparent sudden commencement associated low energy proton increase similar to those reported by Pieper et a1 (1962), Zmuda et al (1963), and Van Allen et al (1962).

Possible discrepancies between the 1963 38C data and the INJUN 4 data may arise from (1) northern and southern polar cap asymmetries, (2) local time (impact zone) effects, and (3) latitude variations across the polar cap. The two southern polar cap points from 1963 38C (February 6, 0539 UT and 0732 UT) do not display any significant norihsouth asymmetry a1though Krimigis and Van Allen (1967) indicate that such an asymmetry may exist. Local time effects, while unknown, could be important as the two satellites are $\sim 7-11$ hours apart in local time. 
A good example of apparent latitude variations across the polar cap occurs during the 1963 38C pass of 0232 UT, February 6, and is shown in Figure 3. This pass occurs near the peak of the first intensity maximum of low energy polar cap protons. In Figure 3 are shown the count rates of an integral electron spectrometer channel which has a threshold at $280 \mathrm{kev}$ (Williams and Smith, 1965) and of the proton spectrometer channel sensitive to protons in the range $2.2-8.2 \mathrm{Mev}$. Both detectors are aligned with their look directions normal to the local line of force. The proton channel shows a response which rises to a plateau value of about $1 \mathrm{cps}$ at $\mathrm{L} \simeq 8\left(\Lambda=67.5^{\circ}\right)$, remains essentially constant out to $\mathrm{L} \sim 40\left(\Lambda=80^{\circ}\right)$, and then increases suddenly by a factor of $\sim 3$. Comparison with the electron spectrometer channel which shows an outer zone trapped electron profile dropping to cosmic ray background levels at $\mathrm{L} \sim 10$, indicates that electron contamination in the proton channel is negligible.

To more clearly illustrate the low energy proton intensity increase, the response of an omnidirectional detector sensitive to protons $\geq 2 \mathrm{Mev}$ and electrons $\geq 250 \mathrm{kev}$ is shown in Figure 4. As indicated above, electron contamination is negligible for $L \geq 10\left(\Lambda \geq 71.5^{\circ}\right)$. The normal background count rate for this detector is $\sim 0.24 \operatorname{cps}$ for $\Lambda \geq 72^{\circ}$. The open circles in Figure 4 represent the data points taken from the descending portion of the pass. Unfortunately, not enough data are available to retrace the step at $\sim 80^{\circ}$ and the possibility exists that the increase is temporal rather than spatial. 
The latitude dependence of low energy (1.2 - $2.2 \mathrm{Mev})$ protons over the polar cap has been obtained for passes in which data exists beyond $\Lambda=80^{\circ}$. To improve statistics the count rates from both the $90^{\circ}$ and $180^{\circ}$ low energy (1.2 - $\left.2.2 \mathrm{Mev}\right)$ channels aboard 1963 38C have been averaged. The absence of any significant anisotropies in the low energy proton flux during the event (Bostrom et al, 1967) supports the validity of the above averaging procedure. In addition, $5^{\circ}$ averages in the invariant magnetic latitude, $\Lambda$, were constructed. Figure 5 shows the results of the latitude dependence so obtained for the four available passes.

While a statistically significant enhancement above $\Lambda \simeq 80^{\circ}$ occurs only in the pass of 0232 UT February 6, two of the remaining three passes show an indication of an intensity enhancement at $\Lambda \gtrsim 80^{\circ}$. The only pass displaying a relatively smooth latitude profile over the polar cap is the pass of 1647 UT February 6 , obtained at the maximum of the second low energy proton intensity peak.

Although it is not possible to determine uniquely whether these intensity increases are spatial or temporal in nature, the fact that the three observed increases are all associated with similar high latitudes $\left(\Lambda \gtrsim 80^{\circ}\right)$ indicates that this is a spatial effect. The high latitude intensity increases shown in Figures $3-5$ may be associated with either a high latitude neutral line on the magnetopause or be representative of the first geomagnetic field line interconnecting with the interplanetary field. During the period February 5 - 8, 1965, satellite 1963 38C sampled local times of 1520 - 1530 hours. Thus a 
neutral line (not a point) or interconnection over a wide span of local time is required to explain the above effects observed $\sim 50^{\cup}$ east of the earth-sun line.

Comparison of Polar Cap and Mariner 4 Data: At the time of the event Mariner 4 was located approximately $55 \mathrm{R}_{\mathrm{E}}$ above (north of) the earth-sun 1 ine, $604 R_{E}$ east of the earth-sun 1 ine and $3611 R_{E}$ away from the earth along the earth-sun line in the anti-solar direction. The radius vector from the earth to Mariner 4 made an angle of $\sim 9^{u}$ with the earth-sun line.

The time history of the February 5 event as obtained by the 0.88 4.0 Mev proton channel aboard Mariner 4 along with the normalized polar cap data of Figure 1 are shown in Figure 6. Figure 7 displays a similar time history using the 0.50 - 11 Mev channel of Mariner 4 and the polar cap data of Figure 2. It is seen that the Mariner 4 data also display a complex time structure wherein a succession of intensity maxima are observed. Note that although the times of onset and of maximum intensity in the first peak are the same at both locations within the temporal resolution of the polar cap data, the apparent structure observed over the polar regions early in the event is not observed at the position of Mariner 4.

It is this apparent structure in the low energy proton intensities over the polar cap at the beginning of the event coupled with the simultaneous lack of any similar structure at Mariner 4 which indicates that low energy protons are being alternately allowed and denied access 
to the polar cap regions. This implies a magnetospheric configuration wich varies in concert with changing boundary conditions imposed by the interplanetary medium. While such changes are to be expected, the rapidity of these variations, as implied by the polar cap proton structure, is somewhat surprising (the fastest variation in Figures 1 and 2 is $\sim 30$ minutes.)

In further comparing the low energy proton time histories as observed over the polar cap and at the position of Mariner 4, two approaches have been taken: 1) the time histories as observed over the polar cap and at Mariner 4 are independent and unrelated and the protons simply have a common origin at the sun and 2) the time histories over the polar cap and at Mariner 4 are related and any observed differences in the time histories require explanation. In either of the two considered approaches, the apparent time variations in the polar cap proton data at the beginning of the event imply a changing magnetospheric configuration as described above.

Unrelated Time Histories: Recent results have indicated a filamentary structure to the interplanetary magnetic field with filaments having a spatial diameter much larger than the gyro-radius of, for example, a 10 Mev proton (Bartley et al, 1966; Ness et al, 1966; McCracken and Ness, 1966). The filaments, while being convected through the interplanetary medium by the solar wind, would tend to collimate low energy protons so that they would travel down the length of the filament. 
The time structure observed at Mariner 4 in the low energy proton intensities during the February 5, 1965 event may have been due to a bundle of intertwined filaments being swept past the spacecraft. In the same manner a separate bundle of filaments may have simultaneously swept past the earth producing the time structure observed over the polar regions. Thus the time histories observed at Mariner 4 and over the polar cap would be independent, being caused by two separate bundles of filaments, and would be related only through a common origin for the protons.

However, the agreement in onset time and time of maximum intensity of the first peak at both locations indicates that at least during the first peak, the earth-Mariner observations pertained to a similar interplanetary environment. Either the filamentary structure was large scale compared to earth-Mariner distances or did not vary significantly over earth-Mariner distances.

Following the sudden commencement filament dimensions may be roughly estimated by considering the width of the temporal variations observed at Mariner 4. These widths varied but were in general 22 hours. Using a 2 hour width and a solar wind velocity of $2000 \mathrm{~km} / \mathrm{sec}$ as determined by the sudden commencement on February 6, a filament diameter of $\sim 1.4(10)^{7} \mathrm{~km}$ is obtained. Comparing this with the earth-Mariner distance of $\sim 2.3(10)^{7} \mathrm{~km}$ indicates that the filament structure may have been large scale. However, it still appears possible for two separate filaments to have caused the time histories observed over the polar cap and at Mariner 4. 
Related Time Histories: In this interpretation it is assumed that Mariner 4 was outside of any magnetospheric configuration existing at the time of the February 5, 1965 solar proton event and that the earth-Mariner system samples a similar flux of low-energy protons. The position of Mariner 4 listed earlier is consistent with its being outside any assumed extended tail field configuration. In addition, available evidence is also consistent with this supposition (Van Allen, 1965).

The data in Figures 6 and 7 indicate that the onset time and the time of the first maximum in intensity of low energy protons are roughly the same both over the polar caps and at the position of Mariner. 4. The uncertainty in the time of the polar cap peak allows the relative Mariner 4 - polar cap initial peak times to be consistent with proton transit times (direct transit time from the earth to Mariner 4 for a 1.5 Mev proton is $\sim 27$ minutes). Thus the relative arrival of low energy protons over the polar cap and at Mariner 4 during the first intensity maximum (excluding the previous considerations of the apparent structure early in this peak observed over the polar cap) is consistent with there being significant field line interconnection from the geomagnetic field to the interplanetary field (Dungey, 1961).

As indicated in Figures 6 and 7 , there appear to be two broad peaks, each showing some fine structure, which follow the initial intensity maximum. Making the polar cap-Mariner peak correspondence shown in Figures 6 and 7 , it is apparent that Mariner 4 observes these low energy proton peaks some 3 to 5 hours before they are observed over 
the polar cap. A change in the magnetospheric configuration may have occurred between the first and second intensity maxima such that direct access of low energy protons to the polar cap regions was inhibited and several hours were required for the low energy protons to enter the magnetosphere and arrive over the polar region. The fact that a change in the magnetospheric configuration may have occurred at this time is consistent with the observation of a sudden commencement at 1414 UT February 6, 1965 (Lincoln, 1965). It would not be surprising for the boundary conditions imposed by the interplanetary medium to be changing at that time. However the time of the second Mariner 4 intensity peak, occurring before the sudden commencement, does imply that interplanetary conditions were disturbed $\sim 0.1-0.2$ AU upstream of the shock front.

The 3 - 5 hour delay before low energy protons are observed over the polar cap is suggestive of diffusion effects in the presence of an extended geomagnetic tail (Michel, 1965; Michel and Dessler, 1965). The relative position of the sun, the earth and Mariner 4 excludes the possibility of the delay time being a manifestation of the interplanetary magnetic field lines co-rotating by the earth-Mariner system. The delay times indicated by the data of Figures 6 and 7 yield, assuming a $1.5 \mathrm{Mev}$ proton energy, tail length estimates of $0.3-0.6 \mathrm{AU}$.

Rather than visuali, ing an idealized extended tail configuration in which the earth's field lines extend backwards away from the earth in a smooth and uniform manner, it is perhaps more realistic to consider 
that the earth's field lines break up into a filamentary structure as they are dragged back from the earth by the solar wind. The geomagnetic filaments could become entwined with the interplanetary magnetic field filaments and thus might lead to subsequent particle diffusion across filaments, yeilding delay times similar to those in Figures 6 and 7 . The polar cap-Mariner 4 comparisons later in the event (Figures 6 and 7 ) are less clear.

The fact that the peaks observed over the polar caps are no wider than those observed at Mariner 4 may indicate that a diffusion type process does not occur. Instead, particles may directly and "easily" enter the magnetosphere at some distance from the earth. The observed delay times again yield an access region $\sim 0.4$ AU away from the earth, assuming a $1.5 \mathrm{Mev}$ proton energy.

Two approaches have been described to explain the relative time histories during the February 5, 1965 solar proton event of low energy protons as observed over the polar cap and at Mariner 4 some $3700 \mathrm{R}_{\mathrm{E}}$ downwind of the earth. With the data at hand, it is not possible to choose between the two approaches. In either case, apparent time structure observed in the initial phase of the event over the polar cap and the absence of similar structure at Mariner 4 give evidence for a magnetospheric configuration which varies as its external boundary conditions vary.

Comparisons of absolute fluxes of low energy protons observed over the polar regions and at Mariner 4 show the intensities are nearly the same at both points of observation (Bostrom et a1, 1967). 


\section{SUMMARY AND DISCUSSION}

The following items summarize the observations of low energy $(\sim 1$ Mev) protons during the February 5, 1965 solar proton event, utili,ing data obtained from satellites 1963 38C and INJUN 4 and spaceprobe Mariner 4 .

1. An enhancement of low energy ( $\sim 1$ Mev) proton intensities has been observed at an invariant latitude of $>=80^{\circ}$. Such an enhancement has been observed in 3 of 4 available cases (Figure 5) and implies a spatial rather than a temporal phenomenon. This effect may be a manifestation of the high latitude neutral line on the magnetopause or an indication of the first geomagnetic field line to interconnect with the interplanetary field. The observation of this enhancement $\sim 53^{\smile}$ east of the earth-sun line requires a neutral line or interconnection over a wide span of local time.

2. Polar cap averages of the low energy proton intensities during the event show an apparent rapid and marked structure during the initial rise to maximum intensities (Figures 1 and 2). The lack of structure of comparable amplitude at the position of Mariner 4 (Figures 6 and 7 ) indicates that direct access of low energy protons to the polar regions was being alternately allowed and denied. This further indicates a magnetospheric configuration which varies according to variations in the boundary conditions imposed by the solar wind.

3. Following the initial rise of the event, low energy protons observed at the position of Mariner 4 exhibited a complex temporal behavior similar to that observed over the polar regions. The distance 
of Mariner 4 from the earth $\left(\sim 3700 \mathrm{R}_{\mathrm{E}}\right.$ in nearly the anti-solar direction) precludes placing a unique interpretation on the comparison of the lowenergy proton behavior over the polar cap and at Mariner 4. The relative time histories may be explaned by (1) the passage of independent . filament bundles past the earth and Mariner 4 or (2) diffusion (or access) into a geomagnetic tail some $0.3-0.6 \mathrm{AU}$ in length. In (1) the time histories over the polar cap and at Mariner 4 are unrelated and only share a common source (sun). In (2), the time histories are assumed related and apparent phase differences are consistent with the above tail length estimates.

of further interest in the behavior of low energy protons during the February 5, 1965 event is their arrival time in the vicinity of the earth and the subsequent rise to the initial maximum. From Figures 6 and 7 it is seen that low energy protons arrive in the vicinity of the earth shortly ( $\leqslant 3$ hours) after maximum flare intensity.

Figure 8 shows two ecliptic projection of the interplanetary magnetic field assuming a gross overall Archemedian spiral configuation (Parker, 1958). The field line connecting to the flare region is shown as a dashed line and the position of the earth-Mariner 4 system is indicated. Figure 8 a shows conditions at the start of the event assuming a solar wind velocity of $400 \mathrm{~km} / \mathrm{sec}$. Figure $8 \mathrm{~b}$ shows conditions 8 hours later at the time when the initial low energy proton intensities are near their peak. A plasma front velocity of 2000 $\mathrm{km} / \mathrm{sec}$ was used, based on the observed sudden commencement at 1414 UT February 6, 1965. Al1 longitudes refer to the solar surface. The 
eight hour time lapse has been taken into account in Figure $8 \mathrm{~b}$.

Figure 8 indicates that if low energy protons are indeed confined to travel along interplanetary tubes of force, (Bartley et al, 1966; Ness et a1, 1966; McCracken and Ness, 1966) they should have rapid access to near earth regions only for flares occuring in the vicinity of $57^{\mathrm{W}}$ longitude (assuming a $400 \mathrm{~km} / \mathrm{sec}$ solar wind velocity). The present observation of low energy solar flare protons shortly after the event which occurred at $25^{\mathrm{W}}$ longitude indicates that these protons are able to rapidly move some $30^{\circ}$ in longitude either at or near the solar surface (Reid, 1964) or in the region of the shockfront. 


\section{ACKNOWLEDGMENTS}

We acknowledge and deeply appreciate the generosity of Drs.

S. M. Krimigis and J. A. Van Allen of the University of Iowa in making the INJUN 4 and Mariner 4 data available to us. We further acknowledge many stimulating and fruitful discussions concerning these analyses and interpretations with Drs. Krimigis and Van Allen. Constructive discussions with Drs. A. J. Dessler, W. N. Hess, N. F. Ness and E. C. Ray are a1so acknowledged and appreciated.

This research was supported in part by the National Aeronautics and Space Administration under DPR No. R21-009-004(13), and in part by the Nava1 Ordnance Systems Command, Jepartment of the Navy, under contract NOw 62-0604-c. 


\section{REFERENCES}

Bartley, W. C., R. P. Bukata, K. G. McCracken, and U. R. Rao, "Anisotropic cosmic radiation fluxes of solar origin," J. Geophys. Res., 71, 3297-3304, 1966 .

Bostrom, C. O., J. W. Kohl, and D. J. Williams, "The February 5, 1965 solar proton event: 1 . Time history and spectrums observed at 1100 km," J. Geophys. Res. , 72, 1967.

Dungey, J. W., "Interplanetary magnetic field and the auroral zones," Phys. Rev. Letters, 6, 47-48, 1961.

Krimigis, S. M., And J. A. Van Allen, "Observations of geomagnetically trapped protons with INJUN 4," Trans. Am. Geophys. Union, 46, 140, 1965.

Krimigis, S. M., and J. A. Van Allen, "Observations of the solar particle event of 5 - 12 February 1965 with Mariner 4 and INJUN 4", I. Geophys. Res. , 72, 1967

Lincoln, J. V., "Geomagnetic and solar data," J.Geophys. Res., 70, 5953-5955, 1965.

McCracken, K. G., and N. F. Ness, "The collimation of cosmic rays by the interplanetary magnetic field," J. Geophys. Res., 71, 3315$3318,1966$.

Michel, F. C., "Effect on the magnetospheric tail on cosmic ray cut-offs," Planetary Space Sci., 13, 753-760, 1965.

Michel, F. C., and A. J. Dessler, "Physical significance of inhomogeneities in polar cap absorption events," J. Geophys. Res., 70 , 4305-4311, 1965.

Ness, N. F., C. S. Scearce, and S. Cantarano, "Preliminary results from the Pioneer 6 magnetic field experiment," J. Geophys. Res., 71 , $3305-3313,1966$.

Parker, E. N., "Dynamics of the interplanetary gas and magnetic fields," Astrophys. J., 128, 664-676, 1958 .

Pieper, G. F., A. J. Zmuda, C. O. Bostrom, and B. J. O'Brien, "Solar protons and magnetic storms in July 1961," J. Geophys. Res., 67, 4959-4981, 1962 .

Reid, G. C., "A diffusive model for the initial phase of a solar proton event," J. Geophys. Res., 69, 2659-2667, 1964. 
Solar-Geophysical Data, CRPL-F 247 Part B, Centra1 Radio Propagation Laboratory, Boulder, Colorado, March, 1965.

Van Allen, J. A., "Absence of 40-kev electrons in the earth's magnetospheric tail at 3300 earth radii," J. Geophys. Res., 70, 4731$4739,1965$.

Van Allen, J. A., and S. M. Krimigis, "Impulsive emission of $~ 40-\mathrm{kev}$ electrons from the sun," J. Geophys. Res., 70, 5737-5751, 1965.

Van Allen, J. A., W. G. V. Rosser and W. A. Whelpley, "INJUN 1Explorer 12 observations of solar cosmic rays, September 28 to October 4, $1961, "$ Trans. Am. Geophys. Union, 43, 216, 1962.

Whelpley, W. A., "INJUN 4 satellite, spacecraft and mission," Trans. Am. Geophys. Union, 46, 140, 1965.

Williams, D. J., and A. M. Smith, "Daytime trapped electron intensities at high latitudes at 1100 kilometers," J. Geophys. Res., 70, 541-556, 1965.

Zmuda, A. J., G. F. Pieper and C. O. Bostrom, "Solar protons and magnetic storms in February 1962," J. Geophys. Res., 68, 1160-1165, 1963. 


\section{FIGURE CAPTIONS}

Figure 1:

Figure 2:

Figure 3:

Figure 4:

Figure 5:

Figure 6:
Normalized low energy proton intensities averaged over the polar regions during the February 5, 1965 solar proton event. The data are from the $1.2-2.2$ Mev channel of $196338 \mathrm{C}$ and the 0.9 - $2.1 \mathrm{Mev}$ channe 1 of INJUN 4. The flare of February 5 and the sudden commencement of February 6 are indicated.

Same as Figure 1 except that the data are from the $1.2-2.2 \mathrm{Mev}$ channe 1 of $196338 \mathrm{C}$ and the $0.52-4.2$ Mev channel of INJUN 4 .

Response of electron spectrometer channel 1 ( $\mathrm{E}_{\mathrm{e}} \geq 280$ kev) and proton spectrometer channel $2\left(2.2 \leq \mathrm{E}_{\mathrm{p}} \leq 8.2\right.$ Mev) over the polar cap during the 1963 38C pass of 0232 UT February 6, 1965. The data show an enhancement of protons at high $\mathrm{L}$ values (latitudes). The folding over of the curve at high $L$ values reflects the descending portion of the pass.

Response of omnidirectional channel A ( $E_{e} \geq 280 \mathrm{kev}$, $E_{p} \geq 2.0 \mathrm{Mev}$ ) over the polar cap for the 1963 38C pass of 0232 UT February 6, 1965. The response of this detector shows very clearly the proton enhancement at high latitudes. The outer zone electron profile is seen to terminate at $\Lambda \simeq 72^{\circ}$. Above this value the background count rate for this detector is $\sim 0.24$ counts per second. Open circles show descending portion of pass.

Low energy proton intensity averages over the polar cap for all available $196338 \mathrm{C}$ passes extending to $\Lambda>80^{\circ}$. All passes are from February 6, 1965. The data are from the lowest energy proton channel of $196338 \mathrm{C}, 1.2 \leq \mathrm{E}_{\mathrm{p}} \leq 2.2 \mathrm{Mev}$. To improve statistics, both proton spectrometers (see text) have been averaged together and five degree averages in $\Lambda$ have been constructed. Three of the four available passes show an enhancement at $\Lambda Z 80^{\circ}$. The pass at 1647 UT, displaying a smooth polar cap profile, occurs at the second low energy proton intensity peak shown in Figures 1 and 2 .

Comparison of low energy proton observations over the polar cap and at Mariner 4 during the February 5, 1965 solar proton event. See text for discussion. 
Figure 7: Same as Figure 6 except for different energy channels.

Figure 8: $\quad$ Ecliptic projection of sun-earth-Mariner 4 system showing Archemedian spiral interplanetary field configuration, (a) at start of flare assuming 400 $\mathrm{km} / \mathrm{sec}$ solar wind velocity and (b) 8 hours after flare using a shock front velocity of $2000 \mathrm{~km} / \mathrm{sec}$ as determined by sudden commencement of February 6 . Dashed line connects to flare. The diagram shows the inaccessibility of low energy protons to the earthMariner 4 region if the protons are confined to the flux tube emanating from the flare region. 


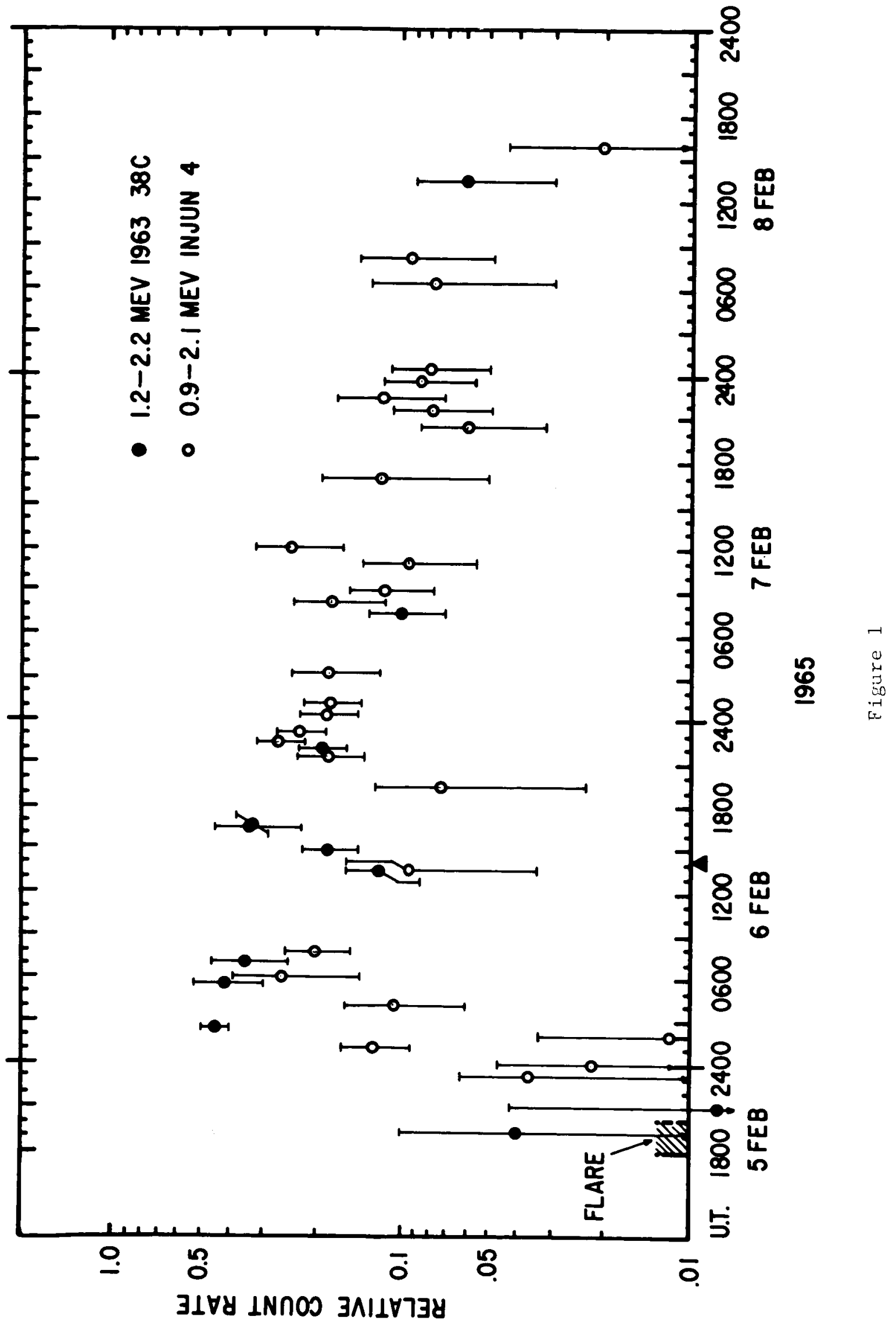




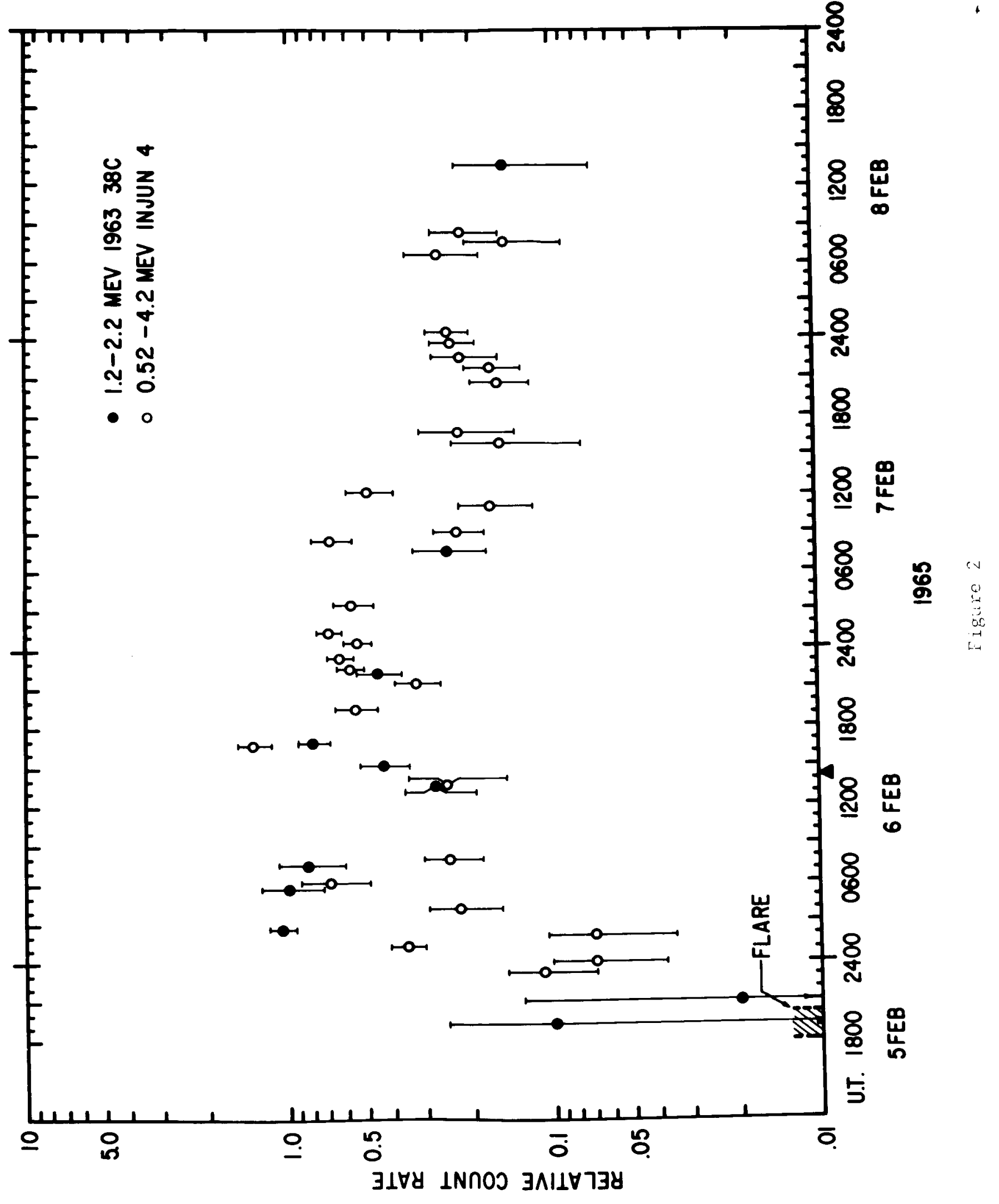




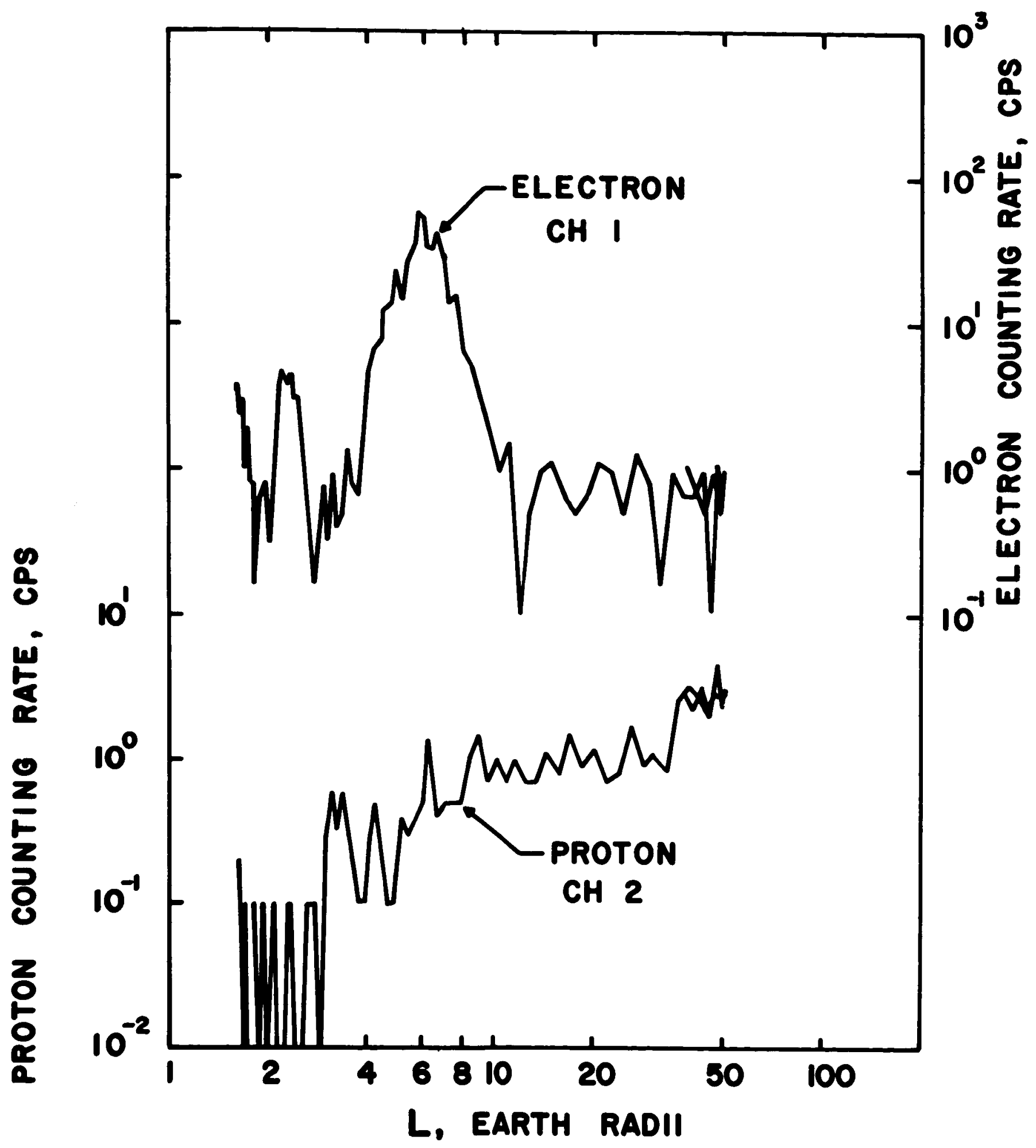

Figure 3 


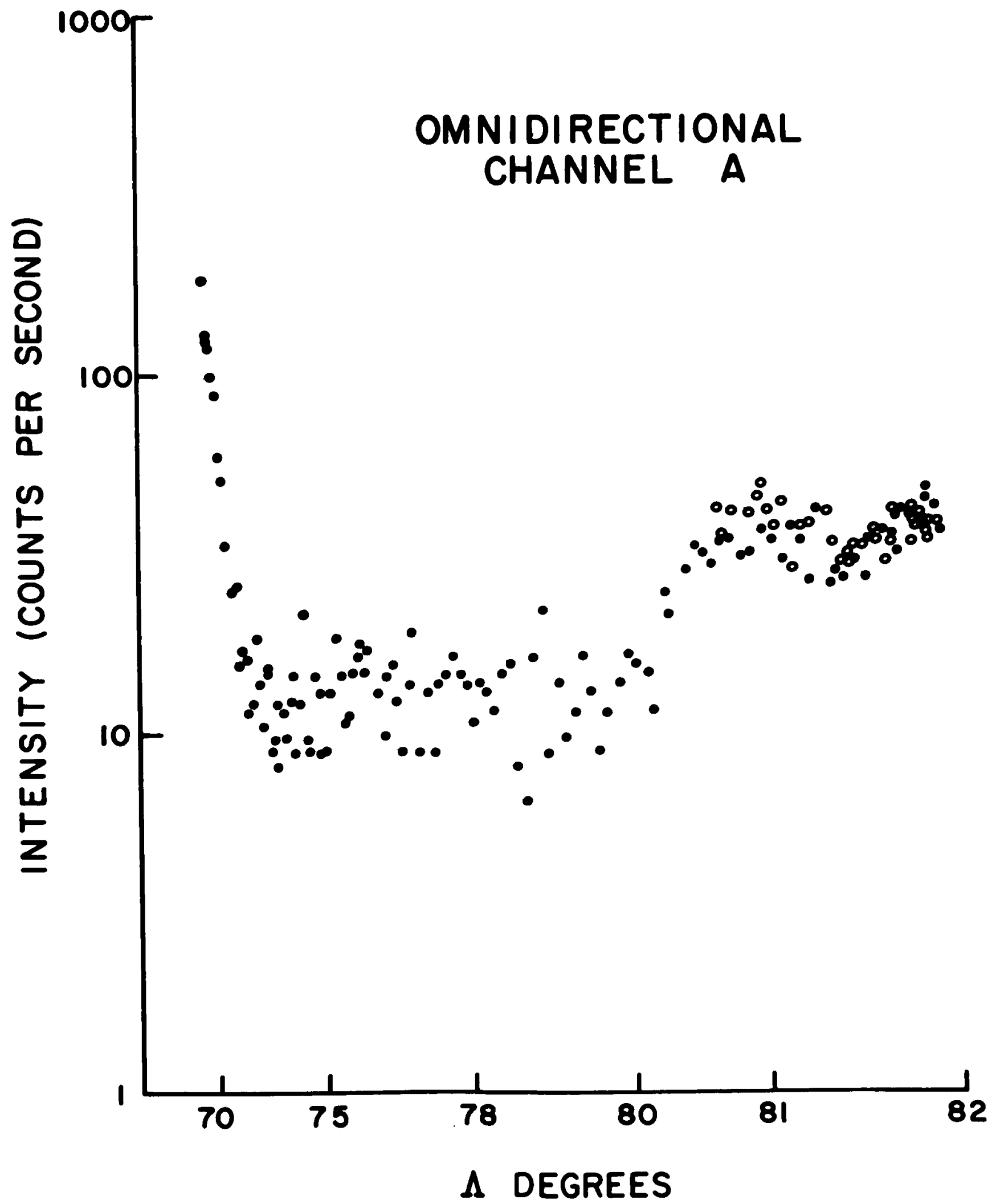

Figure 4 


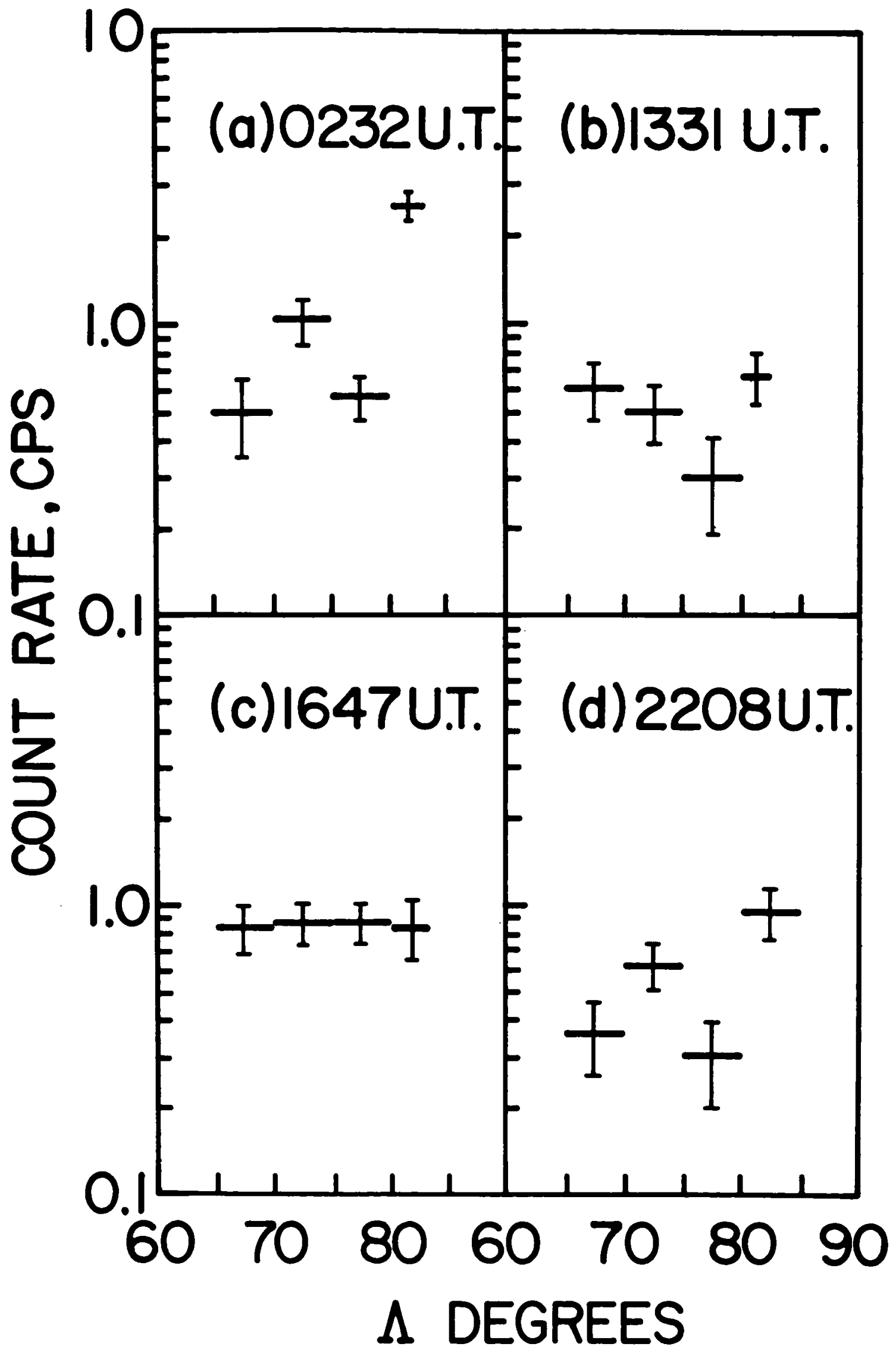

Figure 5 


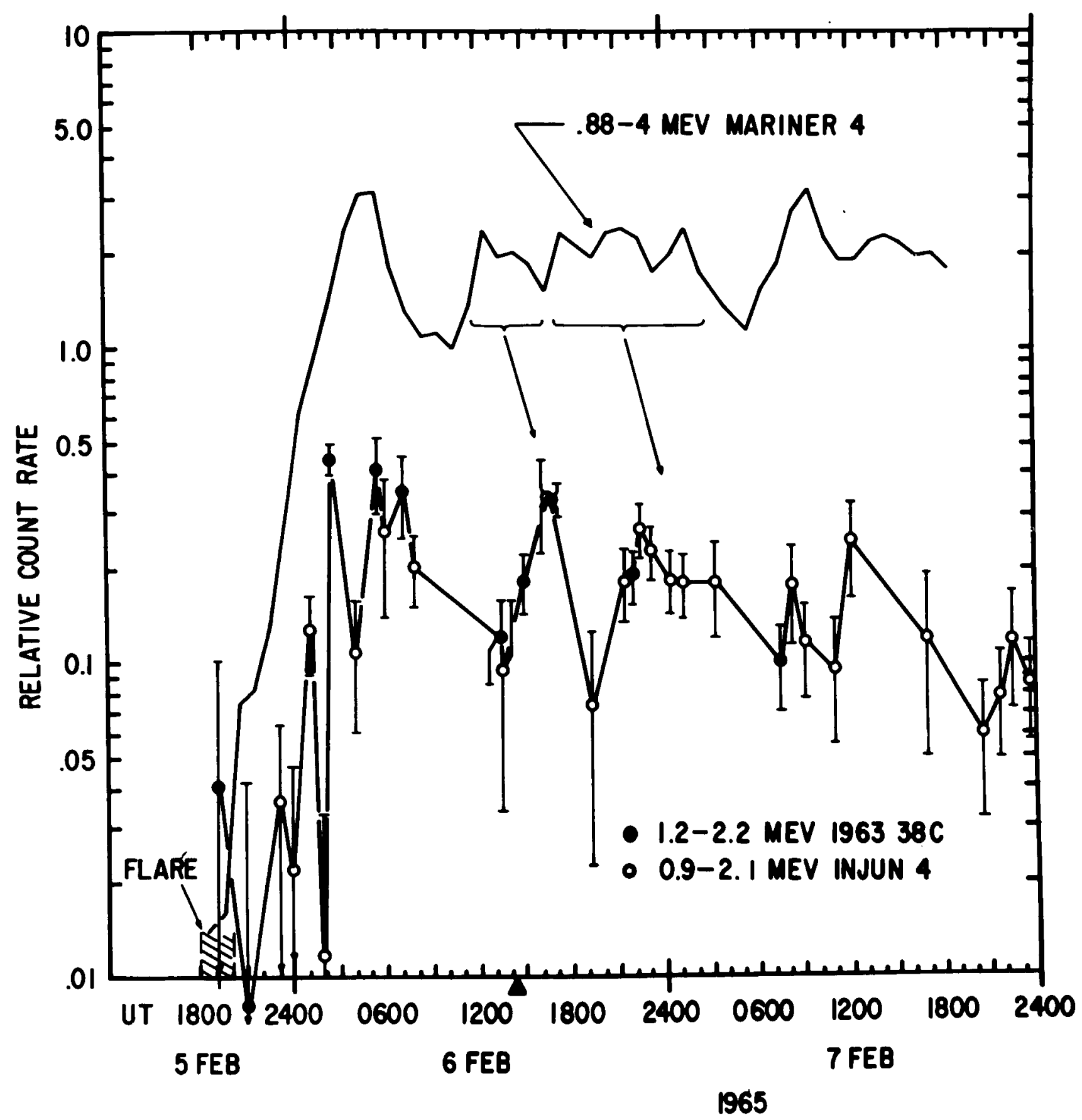

Figure 6 


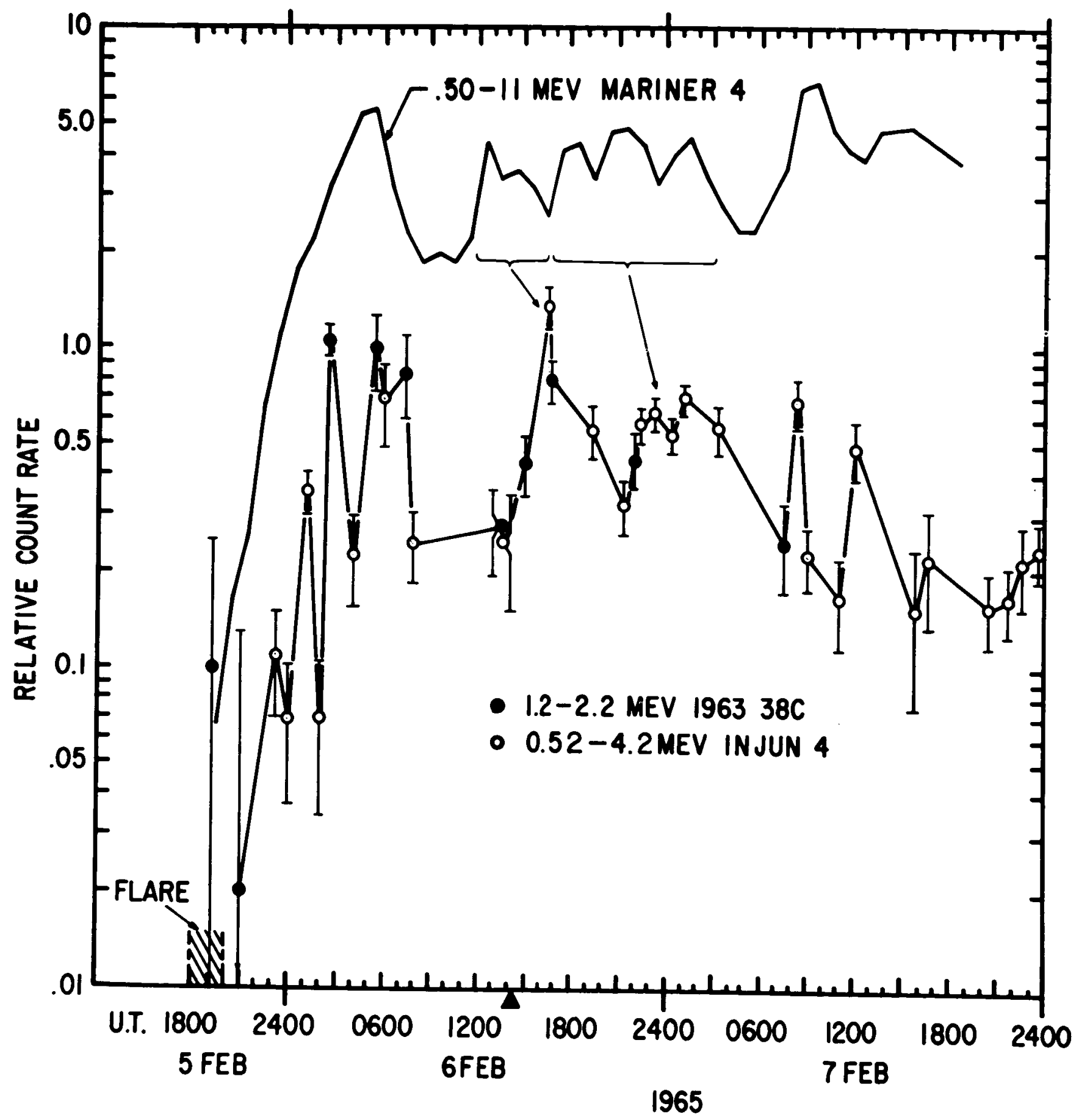

Figure 7 

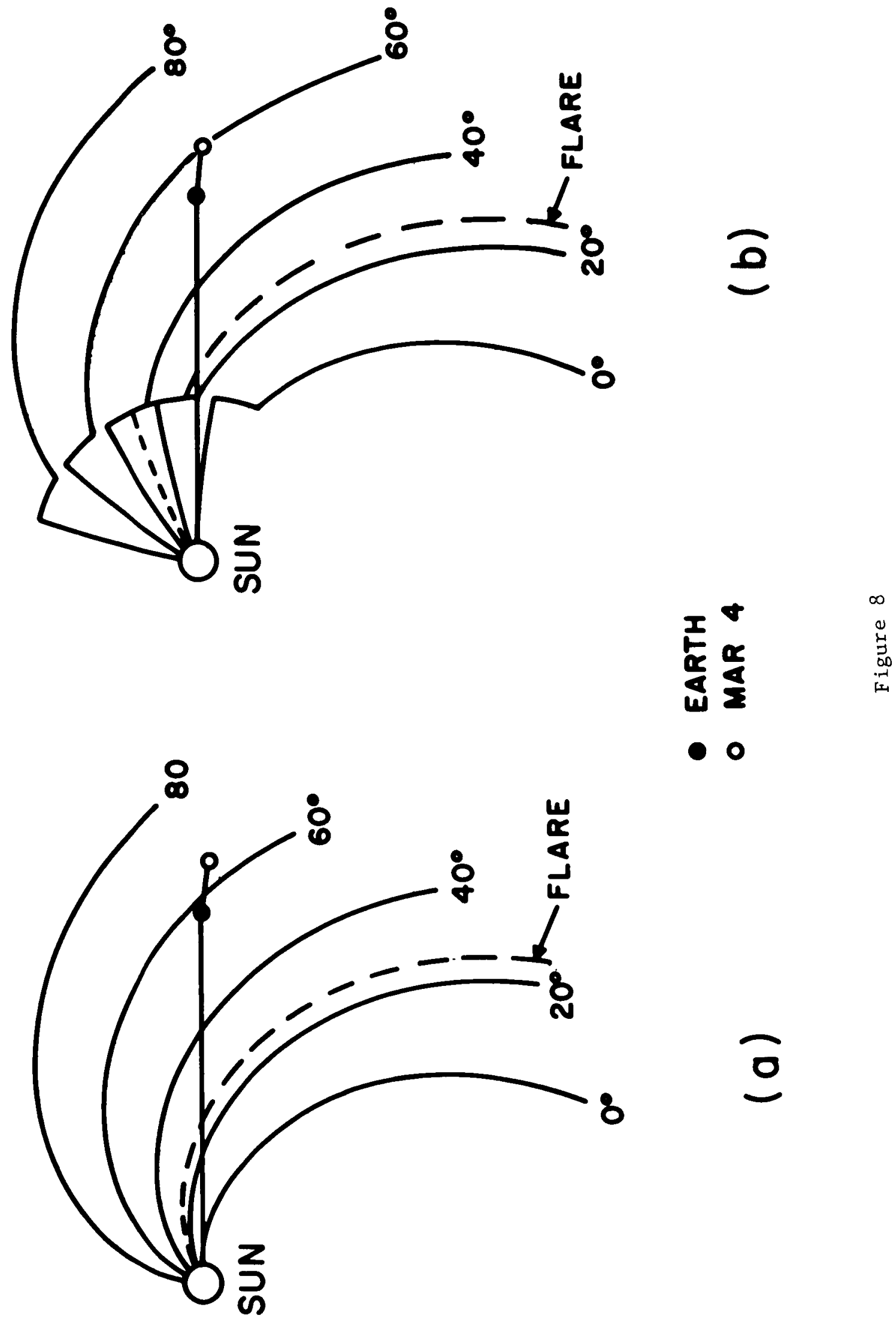

0 